\title{
Detection of Helicobacter pylori using PCR in dental plaque of patients with and without gastritis
}

\author{
Amir Eskandari ${ }^{1}$, Ali Mahmoudpour ${ }^{2}$, Nader Abolfazli ${ }^{1}$, Ardeshir Lafzi ${ }^{3}$ \\ ${ }^{1}$ Department of Periodentics, School of Dentistry, Tabriz University of Medical Sciences, Tabriz, Iran \\ ${ }^{2}$ Drug and Applied research center, Tabriz University of Medical sciences, Tabriz, Iran \\ ${ }^{3}$ Department of Periodentics, School of Dentistry, Tabriz University of Medical science, Tabriz, Iran
}

Correspondence:

Department of Periodentics, School of Dentistry

Tabriz University of Medical Sciences

Tabriz, Iran

amirr22@yahoo.com

Received: 09/03/2009

Accepted: 02/08/2009

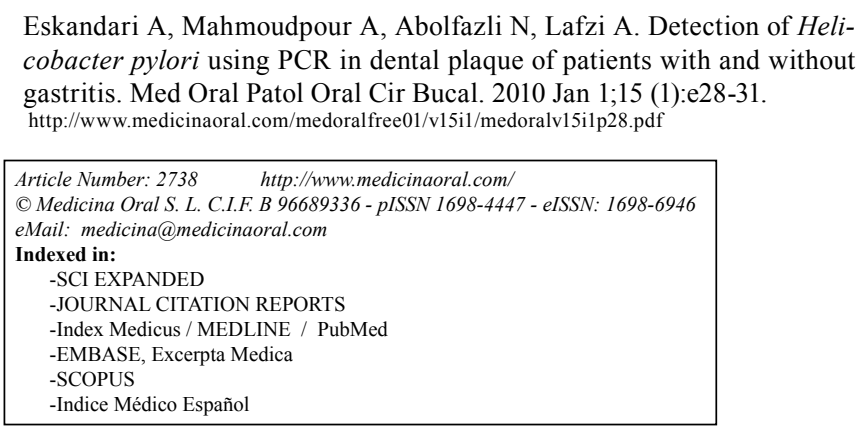

\begin{abstract}
Objectives: Helicobacter pylori (H. pylori) accounts for gastritis, peptic ulcer and is a probable cause of gastric cancer. Since its detection in the oral cavity, concerns have been raised about dental plaque as a reservoir for reinfection. The aim of this study was to detect the organism in the dental plaque and to determine the association, if any, between $H$. pylori gastritis and dental plaque contamination causing $H$. pylori. Study design: A polymerase chain reaction-based method was used for detection of $H$. pylori in clinical specimens. Supra and subgingival samples were collected from 67 patients with chronic periodontitis, 23 of whom were also suffering from gastritis. The data were analyzed with Chi square and Fisher exact test and the statistical significance was set to 0.05 . Results: H. pylori was scarce in patients with periodontitis(5.9\%). There was a significant association between the presence of $H$. pylori in the dental plaque and gastritis $(\mathrm{p}=0.012)$. Conclusions: Although rarely seen, $H$. pylori infected dental plaque may be a source for reinfection. It is therefore suggested that professional plaque removal and oral hygiene procedures be performed, along with the antibiotic treatment of $H$. pylori.
\end{abstract}

Key words: Helicobacter pylori, polymerase chain reaction (PCR), gastritis, dental plaque.

\section{Introduction}

Helicobacter pylori is a fastidious, microaerophilic spiral gram-negative organism that is strongly associated with active and chronic gastritis as well as with peptic and duodenal ulcer diseases (1). H. pylori infections are present worldwide. Although many aspects of the epidemiology of $H$. pylori infection are known, the mode(s) of transmission remains unclear. Recently, $H$. pylori has been found in association with dental plaque, suggesting that the oral environment may be one of the many potential pathways for transmission $(2,3)$.
The detection of $H$. pylori infection in stomach is currently made by rapid urease test, tissue histology or culture, by performing endoscopy. Noninvasive diagnostic tests such as serologic and urea breath tests may also be used to diagnose or monitor eradication of the organism after therapy (4). The presence of cross-reactive antigens between the oral organism Campylobacter rectus and H. pylori may affect the serologic diagnosis of H. pylori infections, especially when saliva is used (5). The use of PCR on biopsy specimens does not add much to other techniques employed for the detection of the 
organism in the stomach $(6,7)$. However, the molecular methods are useful when analyzing specimens, such as gastric juice, environmental samples, oral secretions, and stool samples, for which the regular methods lack sensitivity (8).

There are conflicting results about the role of dental plaque as a source of infection for Helicobacter pylori with a detection rate ranging from $0 \%$ to more than $90 \%$.The current case-control study was undertaken to examine the association between the presences of $H$. pylori in dental plaque with $H$. pylori gastritis. In this study PCR and urease test were used respectively for detection of $H$. pylori in dental plaques and gastric biopsy specimens.

\section{Material and Methods \\ Sampling}

A total of 67 chronic periodontitis patients among patients referring to Periodontics Department of Tabriz Dental School were enrolled in the study after the subjects were informed of the study protocols and they had given informed consent. The study protocol was approved by the ethical committee and research vice chancellor of Tabriz University of Medical Sciences. The inclusion criteria were presence of teeth in the oral cavity, periodontal pocket with a minimum depth of $4 \mathrm{~mm}$ and bleeding on probing, and lack of previous history of reflux. Subjects with diabetes, pregnant women, HIV-positive patients, smokers and patients who had taken antisecretor or antimicrobials within the previous 2 months were excluded from the study.

A questioner was completed for each patient. Dental plaque was removed from the at least 2 teeth surfaces (one anterior and one posterior teeth) with a sterile periodontal curette. Both supra- and subgingival plaques were collected by an upward scrape against the tooth surface. After sampling, probing depth was recorded using William's calibrated periodontal probe. Twenty three of subjects were later diagnosed for H. pylori's gastritis by endoscopy and rapid urease test by a gastroenterologist at the Research Center for Gastroenterology of Tabriz Medical University.

Biopsy specimen were taken from the antrum lesser curvature mucosa $3-4 \mathrm{~cm}$ proximal to the pylorus. The urease test was considered positive when the urea solution changed from yellow to red at room temperature within 6 hours.

\section{Extraction of DNA}

DNA was extracted from dental plaque or strains of Helicobacter pylori by the method described elsewhere (9) with our some minor modifications. Briefly, dental plaque samples were placed into sterile tubes containing $0.5 \mathrm{ml}$ of trypticase soy broth then vortex mixed for 30S. Lysate were prepared for use in PCR by addition of $90 \mathrm{ul}$ of homogenate to $10 \mathrm{ul} 10 \mathrm{X}$ lysis buffer
(100mM Tris-HCL, $\mathrm{pH} 8.0 ; 10$ mm EDTA; Triton X-100 $10 \%$ ) and boiling for 5 minutes. A 5 -ul sample of lysate was used in each PCR reaction and the reminders of the lysates were stored at $-70 \mathrm{C}$.

PCR amplification

Primers JW22 (5-CGTTAGCTGCATTACTGGAGA-3) and JW23 (5-GAGCGCGTAGGCGGGATAGTC-3)targeting the $16 \mathrm{~S}$ ribosomal RNA gene of $H$. pylori were used according to the published data $(9,10)$. PCR Amplification was performed according to the method described by Riggio and Lennon (10). PCR amplication was performed in reaction volume of $50 \mathrm{ul}$ consisting of $5 \mathrm{ul}$ of plaque lysate or $1 \mathrm{ul}$ of $H$. pylori genomic DNA and either 45 or $49 \mathrm{ul}$ of reaction mixture containing $1 \mathrm{x}$ PCR buffer, 1 unit of Taq DNA polymerase, $0.2 \mathrm{mM}$ of each deoxinucleotide triphosphates and each primer at a concentration of $0.2 \mathrm{uM}$. After an initial denaturation step of $95 \mathrm{C}$ for 5 minutes, there were 40 cycles of denaturation steps at $94 \mathrm{C}$ for 1 minute, annealing at $60 \mathrm{C}$ for 1 minutes and extention at $72 \mathrm{C}$ for $1 \mathrm{~min}$, followed by a final extention step at $72 \mathrm{C}$ for 10 minutes. Amplified products were always a single band of the expected size (295bp).

Statistical Analysis

All data double entered into a SPSS, v13 software. Chi-square and Fisher exact test were employed for the analysis of detection frequency of $H$. pylori in dental plaques of patients with and without gastric infection, and the association between the presence of $H$. pylori in dental plaques and $H$. pylori gastritis. In the present study a $\mathrm{p}$ value less than 0.05 was considered to indicate statistical significance.

\section{Results}

A total of 67 patients with a mean age of 42.3(SD: 12.52) participated in this study. Of these patients, 31 were male and 36 were female. (Table 1).

H. pylori was detected in dental plaque specimens of 4/67 patients (5.97\%) by PCR. Dental plaque specimens of 4 patients among a total of $23 \mathrm{H}$. pylori gastritis diagnosed patients' harbored $H$. pylori (17.39\%) while this bacterium was not found in dental plaque samples of other 44 patients with periodontitis but without $H$. pylori gastritis. An association was found between the presence of $H$. pylori in dental plaques and H. pylori gastritis $(\mathrm{p}=0.012)$.

Table 1. The association of helicobacter pylori infection of dental plaque and stomach.

\begin{tabular}{|c|c|c|c|c|}
\hline \multirow{2}{*}{ Sex } & \multicolumn{2}{|c|}{$\begin{array}{c}\text { Helicobacter pylori } \\
\text { in oral cavity }\end{array}$} & \multicolumn{2}{|c|}{$\begin{array}{c}\text { Helicobacter pylori } \\
\text { in stomach }\end{array}$} \\
\cline { 2 - 5 } & Positive & negative & Positive & negative \\
\hline Male & $3(9.7 \%)$ & $28(90.3 \%)$ & $10(32.3 \%)$ & $21(67.7 \%)$ \\
\hline Female & $1(2.8 \%)$ & $35(97.2 \%)$ & $13(36.1 \%)$ & $23(63.9 \%)$ \\
\hline Total & $4(5.9 \%)$ & $63(94.1 \%)$ & $23(34.3 \%)$ & $44(65.7 \%)$ \\
\hline
\end{tabular}




\section{Discussion}

H. pylori infection is one of the most common bacterial infections in man. The infection is widely accepted as an important cause of gastritis and is strongly associated with peptic ulcer disease and gastric cancer The human stomach was considered to be the only reservoir for $H$. pylori until bacteria were discovered in the human dental plaque, in oral lesions or ulcers, in oral cavity, and in saliva (11).

There are conflicting reports about the isolation rate of H. pylori from dental plaques. Detection rate ranging from 0 to $90 \%$ has been reported in different studies. The wide variations in the prevalence of $H$. pylori in the oral cavity probably originate from methodological differences among studies rather than from true geographical variations.

PCR has been found to have higher detection rates of $H$. pylori in gastric biopsy and in microbial dental plaque compared to other identification tests. Among them, in vitro urease tests are dependent on the existence of urease in gastric biopsy specimens as $H$. pylori is the only urease-positive bacteria recovered from stomach. Conversely, $H$. pylori-like organisms were urease, catalase, and oxidase-positive and grew microaerophilically, but they were negative on $H$. pylori-specific PCR analysis, demonstrating the possibility of false identification (12). For this reason, in the present study, urease test was not used for detection of $H$. pylori in microbial dental Plaque because it was considered to be an unreliable test in the identification of $H$. pylori in microbial dental plaque. In this study we detected H. pylori in dental plaques of $5.97 \%$ of periodontitis patients, and our findings showed that there is an association between $H$. pylori of dental plaque and stomach.

In a study by Butt et al. (13), urease test and cytology detected H.pylori in $100 \%$ and $88 \%$ of dental plaque samples respectively. They concluded that the prevalence of $H$. pylori in dental plaque of patients with dyspepsia was very high and indicating it to be a major reservoir of infection. Their conclusion is in agreement with our study but the high prevalence of $H$. pylori in dental plaque could be due to urease-producing organisms which commonly found as part of the normal flora of oral cavity.

Chitsazi et al. (14) based on the results of rapid urease test on oral specimens, reported that H.pylori was detected in $34.1 \%$ of dental plaque specimens. Again this higher prevalence in comparison with our study could be due to urease-producing organisms. Also they did not find any association between H.pylori in dental plaque and stomach which is not in agreement with our study. Martinez-Gomis et al. (15) found no H. pylori in the dental plaque of non-gastritis patients that supports our findings. Gebara et al. (16) reported a high prevalence of H. pylori in the dental plaque, using PCR, which is not in agreement with our low prevalence results but Kignel et al. (17) reported a 5\% prevalence of $H$. pylori in the dental plaque of patients with gastritis and they believed that supragingival dental plaque and saliva may not be relevant reservoirs of $H$. pylori.

Although PCR is a very sensitive and specific method capable of detecting scarce bacterial amounts, its results depends on the primer's specificity and sensitivity. For example in a PCR test for detection of H. pylori in the oral cavity the use of primers related to the bacterial urease activity will be confounding factor. Last but not the least, PCR method for detecting H. pylori in the oral cavity can give result ranging from $0-90 \%$ that can be explained by different ethnic variation and the PCRrelated methodology (18).

Gebara et al. (19) showed persistence of H. pylori in the oral cavity after systemic eradication therapy and concluded mouths of patients with gingivitis or with chronic periodontitis, who are positive for $H$. pylori in their stomachs, may be considered as reservoirs of these bacteria capable of increasing the risk of gastric re-infection in these patients. The mechanism by which H. pylori reaches the oral cavity is unknown. It is possible that the occasional reflux from the gastric reservoir allows colonization of the oral cavity. It is also possible that the reverse is true.

In summary, there is increasing evidence for a role of the oral cavity in transmission of $H$. pylori, and new methods of detection continue to support this (20). However, this subject requires considerably more investigation before definite involvement of the oral cavity can be confirmed and preventive measures can be tailored toward the prevention of oral spread. More specifically, more extensive studies to confirm identical or closely related strains in the mouth and stomach are required. Also, a specific niche for $H$. pylori should be identified in the oral cavity, and the association of H. pylori with other members of the oral microflora needs to be addressed. More successful culturing techniques for oral H. pylori are also needed to confirm its viability and thus infectious status.

\section{Conclusion}

Although the prevalence of the Helicobacter pylori in dental plaque was shown to be very low, but it may be necessary to pay close attention to dental plaques as the possible source of re-infection after treatment of gastritis. In other word, oral cavity may be noted as the second reservoir of Helicobacter pylori colonization. 


\section{References}

1. Graham DY. Helicobacter pylori: its epidemiology and its role in duodenal ulcer disease. J Gastroenterol Hepatol. 1991;6:105-13.

2. Krajden S, Fuksa M, Anderson J, Kempston J, Boccia A, Petrea $\mathrm{C}$, et al. Examination of human stomach biopsies, saliva, and dental plaque for Campylobacter pylori. J Clin Microbiol. 1989;27:1397-8. 3. Majmudar P, Shah SM, Dhunjibhoy KR, Desai HG. Isolation of Helicobacter pylori from dental plaques in healthy volunteers. Indian J Gastroenterol. 1990;9:271-2.

4. Nakamura RM. Laboratory tests for the evaluation of Helicobacter pylori infections. J Clin Lab Anal. 2001;15:301-7.

5. Ishihara K, Miura T, Ebihara Y, Hirayama T, Kamiya S, Okuda K. Shared antigenicity between Helicobacter pylori and periodontopathic Campylobacter rectus strains. FEMS Microbiol Lett. 2001;197:23-7.

6. Lage AP, Godfroid E, Fauconnier A, Burette A, Butzler JP, Bollen A, et al. Diagnosis of Helicobacter pylori infection by PCR: comparison with other invasive techniques and detection of cagA gene in gastric biopsy specimens. J Clin Microbiol. 1995;33:2752-6.

7. Lin SY, Jeng YS, Wang CK, Ko FT, Lin KY, Wang CS, et al. Polymerase chain reaction diagnosis of Helicobacter pylori in gastroduodenal diseases: comparison with culture and histopathological examinations. J Gastroenterol Hepatol. 1996;11:286-9.

8. Westblom TU, Bhatt BD. Diagnosis of Helicobacter pylori infection. Curr Top Microbiol Immunol. 1999;241:215-35.

9. Weiss J, Mecca J, Da Silva E, Gassner D. Comparison of PCR and other diagnostic techniques for detection of Helicobacter pylori infection in dyspeptic patients. J Clin Microbiol. 1994;32:1663-8.

10. Riggio MP, Lennon A. Identification by PCR of Helicobacter pylori in subgingival plaque of adult periodontitis patients. $\mathrm{J}$ Med Microbiol. 1999;48:317-22.
11. Madinier IM, Fosse TM, Monteil RA. Oral carriage of Helicobacter pylori: a review. J Periodontol. 1997;68:2-6.

12. Namavar F, Roosendaal R, Kuipers EJ, De Groot P, Van der Bijl MW, Peña AS, et al. Presence of Helicobacter pylori in the oral cavity, oesophagus, stomach and faeces of patients with gastritis. Eur J Clin Microbiol Infect Dis. 1995;14:234-7.

13. Butt AK, Khan AA, Khan AA, Izhar M, Alam A, Shah SW, et al. Correlation of Helicobacter pylori in dental plaque and gastric mucosa of dyspeptic patients. J Pak Med Assoc. 2002;52:196-200.

14. Chitsazi MT, Fattahi E, Farahani RM, Fattahi S. Helicobacter pylori in the dental plaque: is it of diagnostic value for gastric infection?. Med Oral Patol Oral Cir Bucal. 2006;11:E325-8.

15. Martinez-Gomis J, Diouf A, Lakhssassi N, Sixou M. Absence of Helicobacter pylori in the oral cavity of 10 non-dyspeptic subjects demonstrated by real-time polymerase chain reaction. Oral Microbiol Immunol. 2006;21:407-10.

16. Gebara EC, Pannuti C, Faria CM, Chehter L, Mayer MP, Lima LA. Prevalence of Helicobacter pylori detected by polymerase chain reaction in the oral cavity of periodontitis patients. Oral Microbiol Immunol. 2004;19:277-80.

17. Kignel S, De Almeida Pina F, André EA, Alves Mayer MP, Birman EG. Occurrence of Helicobacter pylori in dental plaque and saliva of dyspeptic patients. Oral Dis. 2005;11:17-21.

18. Nguyen AM, El-Zaatari FA, Graham DY. Helicobacter pylori in the oral cavity. A critical review of the literature. Oral Surg Oral Med Oral Pathol Oral Radiol Endod. 1995;79:705-9.

19. Gebara EC, Faria CM, Pannuti C, Chehter L, Mayer MP, Lima LA. Persistence of Helicobacter pylori in the oral cavity after systemic eradication therapy. J Clin Periodontol. 2006;33:329-33.

20. Young KA, Allaker RP, Hardie JM. Morphological analysis of Helicobacter pylori from gastric biopsies and dental plaque by scanning electron microscopy. Oral Microbiol Immunol. 2001;16:178-81. 